\title{
Effect of Dulaglutide Versus Liraglutide on Glucose Variability, Oxidative Stress, and Endothelial Function in Type 2 Diabetes: A Prospective Study
}

\author{
Hiroe Nagaike $\cdot$ Makoto Ohara Yo Kohata - Munenori Hiromura • \\ Masako Tomoyasu • Michiya Takada - Takeshi Yamamoto • \\ Toshiyuki Hayashi · Tomoyasu Fukui · Tsutomu Hirano
}

Received: November 14, 2018 / Published online: January 5, 2019

(C) The Author(s) 2019

\begin{abstract}
Introduction: To compare the effect of dulaglutide and liraglutide on oxidative stress and endothelial function in patients with type 2 diabetes mellitus (T2DM).

Methods: Twenty-two patients with T2DM who received treatment with liraglutide for at least 12 weeks were randomized to either continue liraglutide or receive dulaglutide for 24 weeks. The primary end points were changes in the diacron-reactive oxygen metabolite (d-ROMs) test, as a marker of oxidative stress, and endothelial function, as determined by the reactive hyperemia index (RHI). The secondary end points were changes in body weight (BW), glucose variability, diabetes treatment satisfaction questionnaire (DTSQ) score, and eating behavior.
\end{abstract}

Enhanced Digital Features To view enhanced digital features for this article go to: https://doi.org/10.6084/ m9.figshare.7485179.

Electronic Supplementary Material The online version of this article (https://doi.org/10.1007/s13300018-0560-8) contains supplementary material, which is available to authorized users.

H. Nagaike · M. Ohara $(\bowtie) \cdot$ Y. Kohata .

M. Hiromura · M. Tomoyasu · M. Takada

T. Yamamoto - T. Hayashi · T. Fukui · T. Hirano Division of Diabetes, Metabolism, and

Endocrinology, Department of Medicine, Showa

University School of Medicine, Tokyo, Japan

e-mail: s6018@nms.ac.jp
Results: There were no significant differences in changes in d-ROMs and logarithmic-scaled RHI (L-RHI) between the two groups after 24 weeks of treatment. Notably, the treatment with dulaglutide was superior to that with liraglutide in terms of mean glucose levels and mean amplitude of glycemic excursions following the 24-week treatment. However, in this regard, the outcome following the treatment with dulaglutide was maintained, whereas that with the treatment with liraglutide was aggravating. The DTSQ score for "convenience" improved in the dulaglutide group. No statistically significant changes in fasting plasma glucose, hemoglobin A1c, and BW were observed between the two groups.

Conclusion: We showed that once-weekly dulaglutide was comparable to once-daily liraglutide in terms of oxidative stress and endothelial function. Switching from liraglutide to dulaglutide improved convenience by decreasing the number of injections without deteriorating glucose metabolism.

Trial Registration: This trial was registered with the University Hospital Medical Information Network (UMIN no. 000034353) on 10 October 2018.

Keywords: Dulaglutide; Endothelial function; Glucose variability; Oxidative stress; Type 2 diabetes mellitus 


\section{INTRODUCTION}

Type 2 diabetes mellitus (T2DM) is a major cause of atherosclerosis and is related to vascular complications [1], while being a major cause of mortality [2]. Therefore, it is important to prevent the development and progression of diabetic complications. A recent study revealed that the use of a glucose-like peptide 1 (GLP-1) receptor agonist (RA) reduces the risk of cardiovascular events in patients with T2DM who are at a high risk of cardiovascular events $[3,4]$.

It has been reported that arteriosclerosis in T2DM patients is caused by an increase in oxidative stress due to glucose variability, which results in vascular endothelial dysfunction [5]. We also reported that the presence of diacronreactive oxygen metabolites (d-ROMs), a surrogate marker of oxidative stress, was associated with daily glucose variability in T2DM patients [6]. Torimoto et al. reported that the reactive hyperemia index (RHI), a marker of vascular endothelial function, as assessed with the EndoPAT $2000^{\circledR}$ system (Itamar Medical, Ltd., Caesarea, Israel), was correlated with daily glucose variability [7]. Therefore, it is considered important to reduce oxidative stress and protect vascular endothelial function for the treatment of T2DM [8].

Recently, our group demonstrated that reducing the frequency of injections, with basal-bolus insulin therapy switched to liraglutide plus basal insulin, improved treatment satisfaction compared with basal-bolus insulin therapy [9].

Dulaglutide derived from human GLP-1 is a once-weekly GLP-1 RA approved in Japan at a dosage of $0.75 \mathrm{mg}$ [10]. In a phase III study, once-weekly dulaglutide $(0.75 \mathrm{mg})$ was not inferior to liraglutide $(0.9 \mathrm{mg}$, the highest available dosage in Japan) for the control of changes in glycated hemoglobin (HbA1c) levels [11]. A recent study reported that patient satisfaction with once-weekly dulaglutide was superior to that of once-daily liraglutide [12]. However, so far no study has demonstrated the differences between the effects of dulaglutide and liraglutide on glucose variability, as calculated by continuous glucose monitoring (CGM).
In addition, there has been no report to date on the suppression of oxidative stress and protective effects on vascular endothelial function in patients with T2DM with the use of onceweekly dulaglutide and once-daily liraglutide.

Therefore, the aim of the present study was to investigate and compare the effects of onceweekly dulaglutide and once-daily liraglutide for 24 weeks on oxidative stress, endothelial function, glucose variability as assessed by CGM, treatment satisfaction, and eating behavior.

\section{METHODS}

\section{Subjects}

Twenty-two patients with T2DM who received liraglutide for at least 12 weeks were randomized to either continue liraglutide $(n=11)$ or dulaglutide $(n=11)$ for 24 weeks. The study was conducted from September 2016 to March 2018. The inclusion criteria were as follows: (1) a diagnosis of T2DM, (2) age $\geq 20$ years, (3) $\mathrm{HbA} 1 \mathrm{c} \geq 6.5 \%$, and (4) treatment with liraglutide for $\geq 12$ weeks. The exclusion criteria were as follows: (1) the use of steroids or anti-inflammatory drugs, (2) diabetic ketosis and coma within 3 months before the start of the study, (3) severe infection, (4) severe trauma or malignancy, (5) an estimated glomerular filtration rate of $<30 \mathrm{ml} / \mathrm{min} / 1.73 \mathrm{~m}^{2}$, according to the Cockcroft-Gault formula, (6) recent surgery, (7) severe liver dysfunction, and (8) pregnancy.

\section{Study Design}

Supplementary Figure S1 summarizes the protocol of this open-label, prospective, and randomized study. Patients were randomly divided into two groups: one that continued treatment with liraglutide and a second that was switched to treatment with dulaglutide. The number of patients withdrawing because of adverse events was similar between both treatment groups (Supplementary Figure S2). At baseline and 24 weeks after continuation of liraglutide or switching to dulaglutide, the following clinical 
and laboratory parameters were measured for all the patients in both groups before breakfast on day 1 of CGM: body weight (BW), low-density lipoprotein cholesterol (LDL-C), high-density lipoprotein cholesterol (HDL-C), triglycerides (TG), estimated glomerular filtration rate, blood pressure, fasting plasma glucose (FPG), and HbA1c content. Plasma oxidant capacity against $\mathrm{N}, \mathrm{N}$-diethylparaphenylenediamine was measured using the d-ROMs test on day 1. CGM was performed for $72 \mathrm{~h}$. The parameters of glucose variability, such as mean glucose level (MGL), percentage coefficient of variation for glucose (\%CV), mean amplitude of glycemic excursion (MAGE), and the mean of daily differences (MODD) in glucose levels were measured on days 2 and 3 . Peripheral vascular arterial tonometry (PAT), as measured with the EndoPAT $2000^{\circledR}$ system, provides noninvasive assessment of the RHI, a marker of vascular endothelial function and vascular health [13]. PAT was measured at baseline and 24 weeks after the start of the study. Because the RHI has a heteroscedastic error structure, L_RHI (a natural logarithm transformation) was used in all the analyses. All the clinical data (i.e., age, sex, smoking habit, and duration of diabetes in years) were retrieved from medical records. No changes were made to the type and dosage of oral hypoglycemic agents, insulin, angiotensin II receptor blocker, angiotensin-converting enzyme inhibitor, and statins during the study period to avoid possible influences on oxidative stress and endothelial function. All the drugs had been prescribed for at least 12 weeks before starting the study.

The Ethics Committee of the Showa University School of Medicine approved the study protocol. The study protocol is in compliance with the Declaration of Helsinki and current legal regulations in Japan. All procedures are in accordance with the ethical standards of the institutional and national committees responsible for human experimentation and with the Helsinki Declaration of 1964, as revised in 2013. Informed consent was obtained from all patients before being included in the study.

\section{Procedures and Measurements}

For each patient, a MiniMed iPro2 CGM system (Medtronic Inc., Northridge, CA, USA) was inserted subcutaneously on day 1 and removed on day 4. To avoid inaccurate results, glucose variability was calculated only on days 2 and 3 . Venous blood samples were drawn for laboratory measurements on day 1 before breakfast. All the patients received a weight-maintaining diet $(25-30 \mathrm{kcal} / \mathrm{kg}$ ideal BW). Glucose variability was calculated using the CGM data. The MGL and incidences of hypoglycemia (percent of $\mathrm{h}$ under $70 \mathrm{mg} / \mathrm{dl}$ in $24 \mathrm{~h}$ ) were measured from the data recorded during CGM and adjusted for self-monitored blood glucose. The MAGE [14] was calculated to assess glucose variability. The MODD [15] was calculated as the mean of the absolute difference between corresponding glucose values on days 2 and 3 . The percentage of $\mathrm{CV}$ was calculated using the coefficient of variation obtained by dividing the standard deviation by the MGL and multiplying by 100 [16].

\section{Laboratory Measurements}

Oxidative stress was measured using the d-ROMs test [17] and a dedicated photometer (F.R.E.E. System; imported by LTD Tokyo from Diacron International s.r.l., Grosseto, Italy), as reported previously [18]. According to the Wismerll kinetic procedure, the change in absorbance/min was expressed as arbitrary units after correction (U.CARR, where 1 U.CARR $=$ the oxidant capacity of a $0.08 \mathrm{mg} / \mathrm{dl}$ of $\mathrm{H}_{2} \mathrm{O}_{2}$ solution; normal range, 250-300 U.CARR). The intra- and inter-assay coefficients of variation were $2.1 \%$ and $3.1 \%$, respectively. An automated analyzer (BM6070; Japan Electron Optics Laboratory Co., Ltd., Tokyo, Japan) was used to measure serum total cholesterol, LDL-C, HDL-C, TG, and creatinine levels. Plasma glucose was measured by the glucose oxidase method, and HbA1c was measured by high-performance liquid chromatography [19]. 


\section{Diabetes Treatment Satisfaction and Eating Behavior}

Treatment satisfaction was assessed using a validated self-administered questionnaire, the diabetes treatment satisfaction questionnaire (DTSQ), at baseline and 24 weeks after the start of the study [20, 21]. The DTSQ is used to assess treatment satisfaction according to eight items that are scored on a 7-point Likert scale from 0 (very dissatisfied) to 6 (very satisfied). The total score was calculated as the sum of scores for the following six satisfaction items: "current treatment," "convenience," "flexibility," "understanding," "recommend," and "continue," yielding a total score from 0 to 36 . The other two items of "perceived frequency of hyperglycemia" and "perceived frequency of hypoglycemia" were treated individually.

Eating behavior was assessed using the questionnaire of The Guideline for Obesity, issued by the Japan Society for the Study of Obesity, at baseline and 24 weeks after the start of the study. As reported previously [22, 23], this questionnaire consists of 55 questions of seven major scales, as follows: (1) recognition of weight and constitution (e.g., "Do you think it is easier for you to gain weight than others?" and "Do you think you gain weight because of less exercise?"), (2) external eating behavior (e.g., "If food smells and looks good, do you eat more than usual?," "If you walk past a supermarket, do you have a desire to buy something delicious?," and "If you see others eating, do you also have the desire to eat?"), (3) emotional eating behavior (e.g., "Do you have a desire to eat when you are irritated?" and "Do you have a desire to eat when you have nothing to do?"), (4) sense of hunger (e.g., "Do you get irritated when you feel hungry?" and "Do you often regret that you have eaten a lot of food?"), (5) eating style (e.g., "Do you eat quickly?" and "Are you known to eat a lot of food?"), (6) food preference (e.g., "Do you often snack on bread?," "Do you like meat?," and "Do you like noodles?"), and (7) regularity of eating habits (e.g., "Is your dinner time too late at night?" and "Do you gain body weight during holidays?"). All the items were rated on a four-point scale ranging from 1 (seldom) to 4 (very often).

\section{End Points and Assessments}

The primary end points were d-ROMs and L_RHI change from baseline after 24 weeks of treatment. The secondary end points were changes in BW, glucose variability, lipid metabolism, DTSQ score, and eating behavior score.

\section{Statistical Analysis}

All normally distributed continuous data sets, as determined by the Shapiro-Wilk test, are expressed as the mean \pm standard deviation. Differences in continuous variables between the liraglutide and dulaglutide groups at baseline and after treatment were evaluated using the independent sample $t$ test or Mann-Whitney $U$ test. The chi-square test was used to compare categorical variables. Pearson's simple linear regression was used to calculate the correlation coefficients. A probability $p$ value $<0.05$ was considered statistically significant. IBM SPSS, version 22, for Windows (IBM Corp., Armonk, $\mathrm{NY}$, USA) was used to perform the analyses.

\section{RESULTS}

\section{Clinical Characteristics}

Of the 27 participants who were enrolled, 22 $(81.5 \%)$ had completed the protocol. Table 1 shows the clinical and laboratory characteristics of the 22 participants (11 participants in the dulaglutide and liraglutide groups each). For the dulaglutide group, the mean age, body mass index, duration of diabetes, and HbA1c content were $\quad 69.2 \pm 9.1$ years, $\quad 26.7 \pm 4.4 \mathrm{~kg} / \mathrm{m}^{2}$, $14.5 \pm 8.9$ years, and $7.1 \% \pm 0.5 \%$, respectively. The baseline characteristics of the patients in the liraglutide group were similar to those in the dulaglutide group. The values of FPG, MGL, MAGE, MODD, \%CV, and hypoglycemia were similar in both groups. Oxidative stress, as measured by the d-ROMs test, and endothelial function, as measured by L_RHI, were similar between groups. 
Table 1 Clinical characteristics of subject at baseline

\begin{tabular}{|c|c|c|c|}
\hline & Dulaglutide $(n=11)$ & Liraglutide $(n=11)$ & $p$ value \\
\hline Age (years) & $69.2 \pm 9.1$ & $67.8 \pm 9.5$ & 0.718 \\
\hline Sex (male) & $9(81.8)$ & $4(36.4)$ & 0.076 \\
\hline Body weight (kg) & $68.3 \pm 13.4$ & $63.4 \pm 12.9$ & 0.401 \\
\hline BMI $\left(\mathrm{kg} / \mathrm{m}^{2}\right)$ & $26.7 \pm 4.4$ & $25.9 \pm 3.6$ & 0.932 \\
\hline Duration of diabetes (years) & $14.5 \pm 8.9$ & $13.7 \pm 8.2$ & 0.844 \\
\hline Hypertension & $10(90.9)$ & $10(90.9)$ & 1.000 \\
\hline Dyslipidemia & $11(100)$ & $10(90.9)$ & 0.748 \\
\hline \multicolumn{4}{|l|}{ Blood pressure (mmHg) } \\
\hline Systolic & $137.5 \pm 17.5$ & $138.5 \pm 19.1$ & 0.938 \\
\hline Diastolic & $78.0 \pm 12.0$ & $80.0 \pm 13.7$ & 0.768 \\
\hline HDL-C (mg/dl) & $48.7 \pm 9.6$ & $50.1 \pm 11.5$ & 0.970 \\
\hline LDL-C (mg/dl) & $84.8 \pm 21.2$ & $81.5 \pm 21.4$ & 0.617 \\
\hline Triglyceride (mg/dl) & $106.8 \pm 23.5$ & $112.5 \pm 41.0$ & 0.318 \\
\hline $\mathrm{eGFR}\left(\mathrm{ml} / \mathrm{min} / 1.73 \mathrm{~m}^{2}\right)$ & $71.5 \pm 18.0$ & $76.6 \pm 19.2$ & 0.900 \\
\hline $\mathrm{FPG}(\mathrm{mg} / \mathrm{dl})$ & $141.5 \pm 27.1$ & $157.0 \pm 58.7$ & 0.156 \\
\hline HbAlc (\%) & $7.1 \pm 0.5$ & $7.2 \pm 0.5$ & 0.222 \\
\hline Mean glucose level (mg/dl) & $159.2 \pm 25.8$ & $149.5 \pm 22.9$ & 0.727 \\
\hline \multicolumn{4}{|l|}{ Markers of glucose variability } \\
\hline $\operatorname{MAGE}(\mathrm{mg} / \mathrm{dl})$ & $105.2 \pm 23.4$ & $110.4 \pm 21.9$ & 0.483 \\
\hline MODD (mg/dl) & $27.5 \pm 8.1$ & $32.6 \pm 14.1$ & 0.235 \\
\hline$\% \mathrm{CV}(\mathrm{mg} / \mathrm{dl})$ & $22.6 \pm 4.7$ & $25.3 \pm 3.4$ & 0.776 \\
\hline FCPR (ng/ml) & $2.6 \pm 1.3$ & $1.7 \pm 0.9$ & 0.714 \\
\hline d-ROMs (U.CARR) & $288.1 \pm 35.5$ & $309.5 \pm 44.3$ & 0.333 \\
\hline L_RHI & $0.48 \pm 0.31$ & $0.34 \pm 0.13$ & 0.268 \\
\hline Macroangiopathy & $5(45.5)$ & $2(18.2)$ & 0.300 \\
\hline Neuropathy & $8(72.7)$ & $10(100.0)$ & 0.300 \\
\hline Nephropathy & $5(45.5)$ & $7(63.6)$ & 0.478 \\
\hline Retinopathy & $3(27.3)$ & $5(45.5)$ & 0.478 \\
\hline \multicolumn{4}{|l|}{ Antidiabetic drugs } \\
\hline Sulfonylurea & $1(9.1)$ & $0(0.0)$ & 0.748 \\
\hline Glinide & $0(0.0)$ & $1(9.1)$ & 0.748 \\
\hline Thiazolidine & $1(9.1)$ & $1(9.1)$ & 1.000 \\
\hline$\alpha$-Glucosidase inhibitors & $5(45.5)$ & $1(9.1)$ & 0.151 \\
\hline
\end{tabular}


Table 1 continued

\begin{tabular}{|c|c|c|c|}
\hline & Dulaglutide $(n=11)$ & Liraglutide $(n=11)$ & $p$ value \\
\hline Metformin & $5(45.5)$ & $5(45.5)$ & 1.000 \\
\hline SGLT2 inhibitors & $1(9.1)$ & $2(18.2)$ & 0.748 \\
\hline Insulin & $3(27.3)$ & $8(72.7)$ & 0.076 \\
\hline \multicolumn{4}{|l|}{ Antihypertensive drugs } \\
\hline $\mathrm{ARB}$ or $\mathrm{ACEi}$ & $9(81.8)$ & $7(63.6)$ & 0.478 \\
\hline Calcium channel blocker & $8(72.7)$ & $7(63.6)$ & 0.748 \\
\hline$\alpha$-Blocker & $1(9.1)$ & $0(0.0)$ & 0.748 \\
\hline$\beta$-Blocker & $4(36.4)$ & $1(9.1)$ & 0.300 \\
\hline \multicolumn{4}{|l|}{ Antihyperlipidemic drugs } \\
\hline Statins & $10(90.9)$ & $9(81.8)$ & 0.748 \\
\hline \multicolumn{4}{|l|}{ DTSQ scores } \\
\hline 1. Current treatment & $5.36 \pm 0.67$ & $4.82 \pm 1.08$ & 0.223 \\
\hline 2. Hyperglycemia & $3.36 \pm 2.01$ & $2.45 \pm 1.92$ & 0.288 \\
\hline 3. Hypoglycemia & $1.18 \pm 1.66$ & $1.73 \pm 1.79$ & 0.406 \\
\hline 4. Convenience & $4.55 \pm 1.04$ & $4.09 \pm 1.45$ & 0.476 \\
\hline 5. Flexibility & $4.27 \pm 1.35$ & $4.27 \pm 1.42$ & 0.946 \\
\hline 6. Understanding & $4.64 \pm 1.12$ & $4.27 \pm 1.27$ & 0.424 \\
\hline 7. Recommend & $4.64 \pm 1.29$ & $4.09 \pm 1.58$ & 0.459 \\
\hline 8. Continue & $5.00 \pm 1.10$ & $4.73 \pm 1.27$ & 0.631 \\
\hline Total score of 2 and 3 & $4.55 \pm 3.05$ & $4.18 \pm 2.71$ & 0.617 \\
\hline Total score of $1,4,5,6,7$, and 8 & $28.45 \pm 3.93$ & $26.27 \pm 7.07$ & 0.644 \\
\hline \multicolumn{4}{|l|}{ Eating behavior } \\
\hline Total $(\%)$ & $36.31 \pm 19.13$ & $39.16 \pm 20.67$ & 0.646 \\
\hline
\end{tabular}

Data are expressed as the mean \pm standard deviation

1 U.CARR (arbitrary unit) = oxidant capacity of $0.08 \mathrm{mg} / \mathrm{dl} \mathrm{H}_{2} \mathrm{O}_{2}$ solution

$\% C V$ percentage coefficient of variation for glucose, $A R B$ angiotensin II receptor blocker, $A C E$ angiotensin-converting enzyme inhibitor, $d$-ROMs diacron-reactive oxygen metabolites, $e G F R$ estimated glomerular filtration rate, $F C P R$ fasting C-peptide, FPG fasting plasma glucose, $H b A 1 c$ hemoglobin A1c, $H D L-C$ high-density lipoprotein cholesterol, $L D L-C$ lowdensity lipoprotein cholesterol, $L \_R H I$ the natural logarithmic scale reactive hyperemia index, $M A G E$ mean amplitude of glycemic excursions, $M O D D$ mean of daily difference of blood glucose 
i

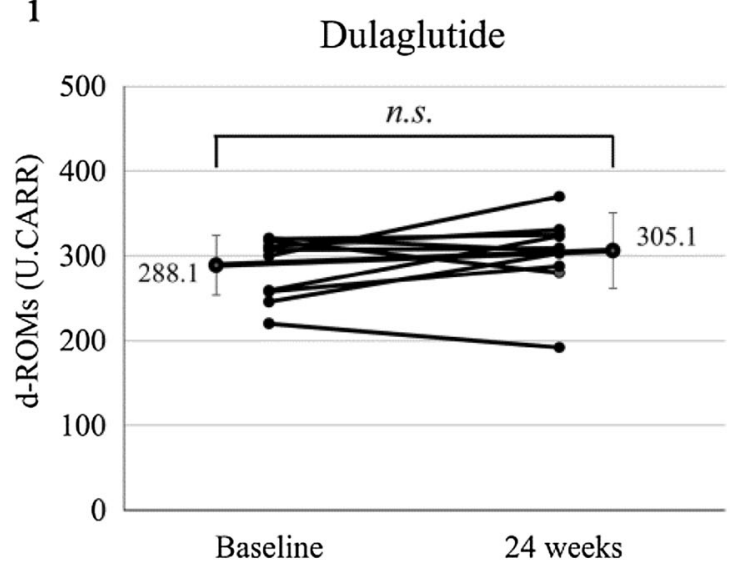

ii

Dulaglutide

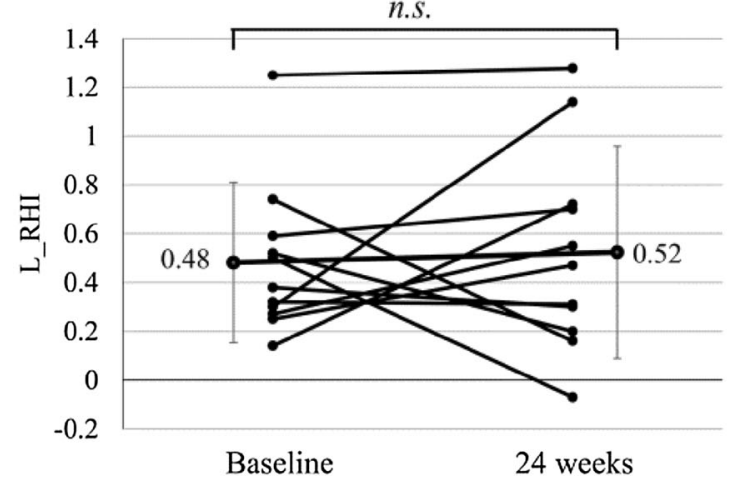

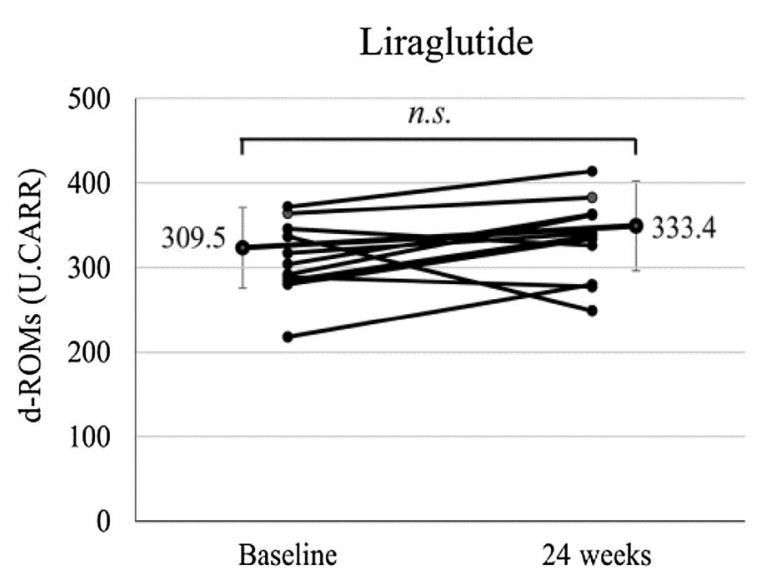

Liraglutide

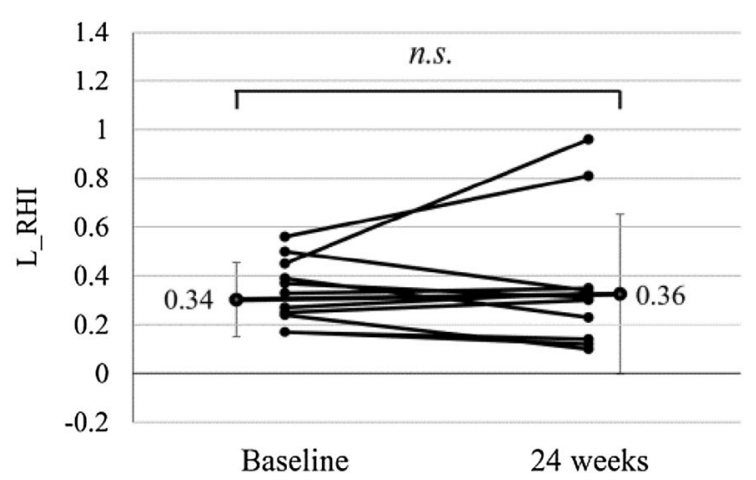

Fig. 1 Comparison of individual change of $d-R O M s$ and L_RHI before and after treatment with dulaglutide versus liraglutide

\section{Effects of 24 weeks of Dulaglutide and Liraglutide on Clinical and Biochemical Parameters}

As Fig. 1 and Table 2 show, there were no significant differences in d-ROMs and L-RHI from baseline to 24 weeks or in the degree of change in d-ROMs and L-RHI between the dulaglutide and liraglutide groups.

Table 2 summarizes the general clinical and biochemical parameters. After 24 weeks of treatment, there were no significant differences in the changes from pre- to post-treatment of clinical and biochemical parameters, with the exception of glucose metabolism, between the dulaglutide and liraglutide groups.

Although there were no significant changes in BW, blood pressure, kidney function, FPG, HbA1c, and FCPR in either group, the change of LDL-C was significantly improved in the liraglutide group compared with the dulaglutide group $(5.18 \pm 8.86$ vs. $-5.09 \pm 11.36$, respectively, $p=0.040$ ).

Figure 2 shows the 24-h blood glucose profiles by CGM. There were no significant differences in the change of MODD and \%CV between the dulaglutide and liraglutide groups $(5.50 \pm 10.40$ vs. $11.03 \pm 22.91, p=0.562$; $-0.73 \pm 3.33$ vs. $2.07 \pm 8.04, p=0.300$, respectively). The dulaglutide group was superior to the liraglutide group in terms of the changes in MGL and MAGE. In particular, regarding the changes in MGL and MAGE, the dulaglutide group remained unchanged, whereas the liraglutide group showed aggravation. $(-3.41 \pm 21.66$ vs. $27.45 \pm 35.34, \quad p=0.023 ; \quad-7.84 \pm 26.10 \quad$ vs. $27.00 \pm 48.28, p=0.034$, respectively). There was no significant difference in the frequency of hypoglycemia between the dulaglutide and liraglutide groups. 
Table 2 Comparison of clinical and biochemical parameters at baseline and 24 weeks

\begin{tabular}{|c|c|c|c|c|c|c|c|}
\hline & \multicolumn{3}{|l|}{ Dulaglutide } & \multicolumn{3}{|l|}{ Liraglutide } & \multirow[b]{2}{*}{$\begin{array}{l}p^{2} \\
\text { value }\end{array}$} \\
\hline & Baseline & 24 weeks & $\begin{array}{l}p^{1} \\
\text { value }\end{array}$ & Baseline & 24 weeks & $\begin{array}{l}p^{1} \\
\text { value }\end{array}$ & \\
\hline Body weight (kg) & $68.3 \pm 13.4$ & $69.1 \pm 14.0$ & 0.898 & $63.4 \pm 12.9$ & $64.4 \pm 13.8$ & 0.857 & 0.949 \\
\hline $\begin{array}{l}\text { Systolic blood pressure } \\
\qquad(\mathrm{mmHg})\end{array}$ & $137.5 \pm 17.5$ & $136.8 \pm 20.2$ & 0.938 & $138.5 \pm 19.1$ & $131.6 \pm 21.1$ & 0.436 & 0.478 \\
\hline $\begin{array}{l}\text { Diastolic blood pressure } \\
(\mathrm{mmHg})\end{array}$ & $78.0 \pm 12.0$ & $79.7 \pm 14.9$ & 0.768 & $79.7 \pm 14.9$ & $76.1 \pm 13.7$ & 0.511 & 0.151 \\
\hline HDL-C (mg/dl) & $48.7 \pm 9.6$ & $50.1 \pm 11.5$ & 0.766 & $50.1 \pm 11.5$ & $49.8 \pm 12.2$ & 0.970 & 0.847 \\
\hline LDL-C (mg/dl) & $84.8 \pm 21.2$ & $90.0 \pm 24.0$ & 0.598 & $81.5 \pm 21.4$ & $76.4 \pm 25.4$ & 0.617 & $0.040^{\mathrm{a}}$ \\
\hline $\mathrm{TG}(\mathrm{mg} / \mathrm{dl})$ & $106.8 \pm 23.5$ & $133.2 \pm 80.3$ & 0.461 & $112.5 \pm 41.0$ & $140.2 \pm 79.0$ & 0.318 & 0.748 \\
\hline $\mathrm{eGFR}\left(\mathrm{ml} / \mathrm{min} / 1.73 \mathrm{~m}^{2}\right)$ & $71.5 \pm 18.0$ & $69.0 \pm 14.2$ & 0.722 & $76.6 \pm 19.2$ & $75.6 \pm 18.7$ & 0.900 & 0.949 \\
\hline FPG $(\mathrm{mg} / \mathrm{dl})$ & $141.5 \pm 27.1$ & $132.8 \pm 33.4$ & 0.509 & $128.3 \pm 23.6$ & $157.0 \pm 58.7$ & 0.156 & 0.101 \\
\hline HbAlc (\%) & $7.1 \pm 0.5$ & $7.1 \pm 0.6$ & 0.970 & $7.2 \pm 0.5$ & $7.6 \pm 0.9$ & 0.222 & 0.116 \\
\hline Mean glucose level (mg/dl) & $159.2 \pm 25.8$ & $155.8 \pm 18.8$ & 0.727 & $149.5 \pm 22.9$ & $177.0 \pm 35.9$ & $0.048^{\mathrm{a}}$ & $0.023^{\mathrm{a}}$ \\
\hline $\operatorname{MAGE}(\mathrm{mg} / \mathrm{dl})$ & $105.2 \pm 23.4$ & $97.4 \pm 27.8$ & 0.483 & $109.9 \pm 21.9$ & $136.9 \pm 42.7$ & 0.087 & $0.034^{a}$ \\
\hline $\operatorname{MODD}(\mathrm{mg} / \mathrm{dl})$ & $27.5 \pm 8.1$ & $33.0 \pm 12.4$ & 0.235 & $32.6 \pm 14.1$ & $43.1 \pm 22.6$ & 0.205 & 0.562 \\
\hline $\begin{array}{l}\text { Hour of under } 70 \mathrm{mg} / \mathrm{dl} \\
(\% 24 \mathrm{~h})\end{array}$ & $0.13 \pm 0.42$ & $0.35 \pm 1.00$ & 0.545 & $0.09 \pm 0.32$ & $0.16 \pm 0.49$ & 0.89 & 0.687 \\
\hline FCPR $(\mathrm{ng} / \mathrm{ml})$ & $2.6 \pm 1.3$ & $2.4 \pm 1.2$ & 0.714 & $1.7 \pm 0.9$ & $2.2 \pm 1.1$ & 0.277 & 0.365 \\
\hline d-ROMs (U.CARR) & $288.1 \pm 35.5$ & $305.1 \pm 44.7$ & 0.333 & $309.5 \pm 44.3$ & $333.4 \pm 48.9$ & 0.244 & 0.562 \\
\hline L_RHI & $0.48 \pm 0.31$ & $0.52 \pm 0.41$ & 0.896 & $0.34 \pm 0.13$ & $0.36 \pm 0.28$ & 0.554 & 0.758 \\
\hline
\end{tabular}

Data are expressed as the mean \pm standard deviation

(1) $p^{1}$ value for the intragroup comparison (pre- vs. post-treatment values in the dulaglutide or liraglutide group; ${ }^{\mathrm{a}} p<0.05$ )

(2) $p^{2}$ value for the intergroup comparison (dulaglutide vs. liraglutide group in the changes from pre- to post-treatment; $\left.{ }^{\mathrm{a}} p<0.05\right)$

$\% C V$ percentage coefficient of variation for glucose, $d$-ROMs diacron-reactive oxygen metabolites, $e G F R$ estimated glomerular filtration rate, FCPR fasting C-peptide, FPG fasting plasma glucose, $H b A 1 c$ hemoglobin Alc, $H D L-C$ highdensity lipoprotein cholesterol, $L_{-} R H I$ the natural logarithmic scale reactive hyperemia index, $L D L-C$ low-density lipoprotein cholesterol, $M A G E$ mean amplitude of glycemic excursions, $M O D D$ mean of daily difference of blood glucose

\section{Effects of Dulaglutide and Liraglutide on DTSQ Status Scores and Eating Behavior}

Table 3 summarizes the self-reported patient treatment satisfaction, as measured by the DTSQ, and eating behavior at baseline at 24 weeks. After 24 weeks of treatment, the sum of the satisfaction-related DTSQ scores for the items of "current treatment," "perceived frequency of hypoglycemia," "flexibility," "understanding," "recommend," and "continue" was unchanged in the dulaglutide and liraglutide groups (from $28.45 \pm 3.93$ to $30.00 \pm 5.60$ and from $26.27 \pm 7.07$ to $26.36 \pm 3.98$, respectively). In the dulaglutide group, the DTSQ 


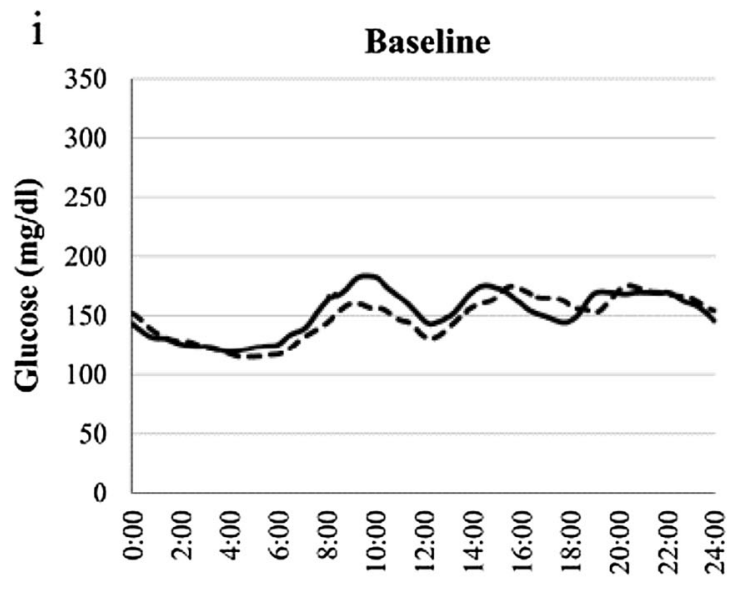

Time of day

-Dulaglutide ---Liraglutide

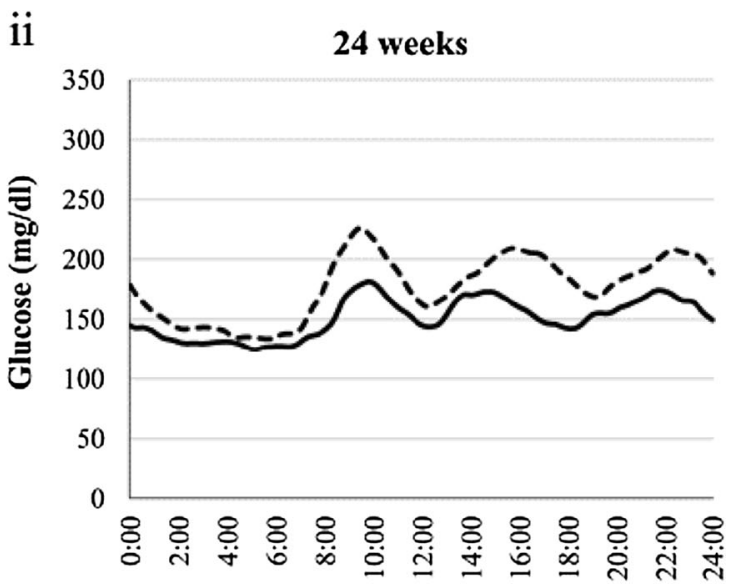

Time of day

-Dulaglutide ---Eiraglutide

Fig. 2 Glucose level over $24 \mathrm{~h}$ during treatment with dulaglutide versus liraglutide at baseline and 24 weeks

score for "convenience" was significantly improved (from $4.55 \pm 1.04$ to $5.45 \pm 0.93$, $p=0.040$ ), while the DTSQ score for "perceived frequency of hyperglycemia" was significantly poorer in the liraglutide group (from $2.45 \pm 1.92$ to $4.18 \pm 1.47, p=0.040$ ). In addition, the DTSQ score for "perceived frequency of hyperglycemia" significantly improved in the dulaglutide group compared with the other group $\quad(-0.45 \pm 2.25$ vs. $1.73 \pm 1.74$, $p=0.034)$.

After 24 weeks of treatment, the eating behavior scores were unchanged in the dulaglutide and liraglutide groups (from $36.31 \pm 19.13$ to $37.11 \pm 19.49$ and from $39.16 \pm 20.67$ to $39.45 \pm 21.05$, respectively).

\section{DISCUSSION}

To the best of our knowledge, the present study is the first to compare the effects of dulaglutide and liraglutide on oxidative stress, vascular endothelial function, glucose metabolism as measured by CGM, patient satisfaction, and eating behavior. The results demonstrated that patients with T2DM who received liraglutide for at least 12 weeks and then switched to dulaglutide showed in the same effect in d-ROMs and L_RHI despite a decrease in the number of injections from once daily to once weekly. These results also demonstrated that dulaglutide improved glucose metabolism and DTSQ scores by decreasing the number of injections from once daily to once weekly compared with liraglutide.

At 26 weeks of the Japanese phase III clinical trial, dulaglutide was not inferior to liraglutide regarding FPG, BW, and HbA1c content change from baseline [24]; however, at 52 weeks, the use of dulaglutide significantly improved the HbA1c content and postprandial plasma glucose both before and after dinner compared with liraglutide [25]. Moreover, HbA1c content was similar between the dulaglutide and liraglutide groups, and the dulaglutide group was superior to the liraglutide group in terms of MGL and MAGE at 24 weeks (at 24 weeks, MGL and MAGE were maintained in the dulaglutide group but were aggravated in the liraglutide group). However, this result may be due to an imbalance in the randomization process regarding T2DM patients with low levels of C-peptide and those receiving insulin therapy in the liraglutide group compared with the dulaglutide group (FCPR, $1.7 \pm 0.92$ vs. $2.6 \pm 1.3, p=0.714$, and insulin therapy, $72.7 \%$ vs. $27.3 \%, p=0.076$, respectively). Jin et al. reported that fasting C-peptide levels were inversely correlated with diurnal daily glucose variability in T2DM patients receiving insulin therapy [26]. Therefore, switching from 
Table 3 Effect of dulaglutide and liraglutide on DTSQs and eating behavior

\begin{tabular}{|c|c|c|c|c|c|c|c|}
\hline & \multicolumn{3}{|l|}{ Dulaglutide } & \multicolumn{3}{|l|}{ Liraglutide } & \multirow[b]{2}{*}{$\begin{array}{l}p^{2} \\
\text { value }\end{array}$} \\
\hline & Baseline & 24 weeks & $\begin{array}{l}p^{1} \\
\text { value }\end{array}$ & Baseline & 24 weeks & $\begin{array}{l}p^{1} \\
\text { value }\end{array}$ & \\
\hline \multicolumn{8}{|l|}{ DTSQ scores } \\
\hline 1. Current treatment & $5.36 \pm 0.67$ & $5.36 \pm 1.21$ & 0.562 & $4.82 \pm 1.08$ & $4.91 \pm 0.54$ & 0.898 & 0.847 \\
\hline 2. Hyperglycemia & $3.36 \pm 2.01$ & $2.91 \pm 1.97$ & 0.606 & $2.45 \pm 1.92$ & $4.18 \pm 1.47$ & $0.040^{\mathrm{a}}$ & $0.034^{\mathrm{a}}$ \\
\hline 3. Hypoglycemia & $1.18 \pm 1.66$ & $1.18 \pm 1.60$ & 0.898 & $1.73 \pm 1.79$ & $1.18 \pm 1.54$ & 0.478 & 0.652 \\
\hline 4. Convenience & $4.55 \pm 1.04$ & $5.45 \pm 0.93$ & $0.040^{\mathrm{a}}$ & $4.09 \pm 1.45$ & $4.36 \pm 1.03$ & 0.699 & 0.171 \\
\hline 5. Flexibility & $4.27 \pm 1.35$ & $5.09 \pm 1.14$ & 0.151 & $4.27 \pm 1.42$ & $4.36 \pm 1.03$ & 1.000 & 0.076 \\
\hline 6. Understanding & $4.64 \pm 1.12$ & $4.18 \pm 1.17$ & 0.365 & $4.27 \pm 1.27$ & $4.36 \pm 0.81$ & 1.000 & 0.217 \\
\hline 7. Recommend & $4.64 \pm 1.29$ & $4.64 \pm 1.03$ & 0.949 & $4.09 \pm 1.58$ & $4.09 \pm 1.64$ & 0.949 & 1.000 \\
\hline 8. Continue & $5.00 \pm 1.10$ & $5.27 \pm 1.19$ & 0.519 & $4.73 \pm 1.27$ & $4.36 \pm 1.03$ & 0.365 & 0.116 \\
\hline Total score of 2 and 3 & $4.55 \pm 3.05$ & $4.09 \pm 3.08$ & 0.699 & $4.18 \pm 2.71$ & $5.36 \pm 1.50$ & 0.116 & 0.217 \\
\hline $\begin{array}{l}\text { Total score of 1, 4, 5, 6, 7, } \\
\text { and } 8\end{array}$ & $28.45 \pm 3.93$ & $30.00 \pm 5.60$ & 0.171 & $26.27 \pm 7.07$ & $26.36 \pm 3.98$ & 0.652 & 0.365 \\
\hline \multicolumn{8}{|l|}{ Eating behavior } \\
\hline Total (\%) & $36.31 \pm 19.13$ & $37.11 \pm 19.49$ & 0.519 & $39.16 \pm 20.67$ & $39.45 \pm 21.05$ & 1.000 & 0.519 \\
\hline
\end{tabular}

Data are expressed as the mean \pm standard deviation

(1) $p^{1}$ value for the intragroup comparison (pre- vs. post-treatment values in the dulaglutide or liraglutide group; ${ }^{a} p<0.05$ )

(2) $p^{2}$ value for the intergroup comparison (changes from pre- to post-treatment in the dulaglutide vs. liraglutide group; $\left.{ }^{\mathrm{a}} p<0.05\right)$

liraglutide to dulaglutide seems superior in terms of glucose metabolism and variability.

Our group recently reported that improvements in glucose variability were associated with therapeutic intervention to reduce oxidative stress [27]. However, despite the significant difference in daily glucose variability in this study, there were no significant differences in oxidative stress and vascular endothelial function between the dulaglutide and liraglutide groups. There are several reasons for this conflicting result. One might be the effect of insulin treatment independent of glucose metabolism. It has been reported that the GLP-1 RA has a direct beneficial effect on oxidative stress and endothelial function, independent of its glucose-lowering effect [28]. However, since the GLP-1 RA was used in both groups, an effect independent of glucose metabolism on oxidative stress and endothelial function was unlikely. Importantly, treatment with insulin tended to be higher in the liraglutide group than in the dulaglutide group in this study. Ceriello et al. reported the cumulative beneficial effects of a combination of insulin and GLP-1 RA on hyperglycemia-induced oxidative stress and endothelial function for patients with T2DM [29]. The antioxidant properties of the two drugs have different mechanisms, where insulin reduces free radical production [30], while GLP-1 RA increases intracellular antioxidant defenses [31]. These mechanisms could explain the fact that the use of a GLP-1 RA and insulin treatment affected oxidative stress and vascular endothelial function characteristics independent of glucose metabolism in the present study. Another reason is that glycemic control was relatively good at baseline; 
therefore, it is possible that $\mathrm{HbA1c}$ and glucose variability could not be remarkably improved. In addition, oxidative stress was close to normal by treatment with liraglutide before the start of the study; thus, oxidative stress could not be improved by treatment with dulaglutide, which may have led to this result.

The results of this study showed that the degree of change in LDL-C was significantly improved in the liraglutide group compared with the dulaglutide group. Although GLP-1 RAs are potent and have potentially beneficial effects on LDL-C [32], the reason for this result is unknown. However, it has recently been reported that liraglutide suppressed PCSK9 expression through an HNF1 $\alpha$-dependent mechanism and decreased LDL receptor, possibly via PCSK9-independent pathways in $\mathrm{db} / \mathrm{db}$ mice [33], which may be related to this result.

In addition to oxidative stress, these results showed additional benefits of switching to dulaglutide from liraglutide on endothelial function, glucose metabolism, and treatment satisfaction. In this study, the total DTSQ scores were similar between the liraglutide and dulaglutide groups. On the other hand, the DTSQ score for the item "hyperglycemia" had significantly increased in the liraglutide continuation group compared with the dulaglutide group. These results were consistent with the significant difference in MGL from pre- to posttreatment in the liraglutide group $(149.5 \pm 22.9$ to $177.0 \pm 35.9$, respectively). Importantly, our study also showed a significant improvement in convenience from pre- to post-treatment in the dulaglutide group. Recently, Takase et al. reported that switching from liraglutide to dulaglutide improved treatment satisfaction, especially regarding convenience and flexibility [12]. Although the data were not shown, in this study, convenience tended to be inversely correlated with the number of injections $(r=-0.552 ; p=0.078)$. Hence, the improved convenience seems to be due to a decrease in the number of injections. However, because the device used for dulaglutide had easier functionality, it possibly contributed to the improved convenience.

There were several limitations to the present study that should be addressed in future research. First, since the study cohort was relatively small, there was no statistically significant difference in terms of the background characteristics of the patients between the two groups. Second, the study lasted only 24 weeks and was not double blinded; therefore, the durability of these benefits is uncertain. Third, this study involved switching from liraglutide to dulaglutide; thus, there is the possibility that the process of switching itself may have brought about a psychologic advantage. Fourth, the dosages of dulaglutide and liraglutide were relatively small, as each was based on the proper dosage for Japanese patients with T2DM. Fifth, considering that the patients had no residual medicine, we believe that strict adherence to the treatment is possible; however, there are some points that we did evaluate accurately. Finally, to deal with these potential issues, the present findings need to be replicated in a longitudinal observation study with a larger population.

\section{CONCLUSION}

Oxidative stress and endothelial function were comparable between once-weekly dulaglutide and once-daily liraglutide. In addition, switching from liraglutide to dulaglutide improved convenience by decreasing the number of injections without deteriorating glucose metabolism.

\section{ACKNOWLEDGEMENTS}

We thank the participants of the study. We thank Chihiro Morisoto, Misaki Hirakawa, Sachiko Sakurai, Sachiyo Mitani, Tomomi Horikawa, and Yasue Moroto of the Showa University School of Medicine for clinical support. We also thank Enago (http://www.enago.jp) for the English language review of this article.

Funding. No funding or sponsorship was received for this study or publication of this article. The article processing charges were funded by the authors. 
Authorship. All named authors meet the International Committee of Medical Journal Editors (ICMJE) criteria for the authorship of this article, take responsibility for the integrity of the work as a whole, and have provided approval for this version to be published.

Authorship Contributions. MO and $\mathrm{HN}$ contributed to the study design, data acquisition, and data analysis and wrote the manuscript. TY, TH, TF, and $\mathrm{TH}$ reviewed and edited the manuscript for intellectual content. MO, $\mathrm{NH}, \mathrm{TH}$, and TF drafted the manuscript. MO, $\mathrm{HN}, \mathrm{YK}, \mathrm{MH}, \mathrm{MT}, \mathrm{MT}, \mathrm{TY}, \mathrm{TH}, \mathrm{TF}$, and TH interpreted the data and critically revised the manuscript. All authors have read and approved the final version of the manuscript.

Disclosures. Tsutomu Hirano has received research funds and lecture fees from Daiichi Sankyo, Eli Lilly, Kowa, Merck Sharp \& Dohme, Mitsubishi Tanabe Pharma, Novo Nordisk Pharma, Ono Pharmaceutical, Sanwa Kagaku Kenkyusho, and Takeda. Hiroe Nagaike, Makoto Ohara, Yo Kohata, Munenori Hiromura, Masako Tomoyasu, Michiya Takada, Takeshi Yamamoto, Toshiyuki Hayashi, and Tomoyasu Fukui have nothing to disclose.

Compliance with Ethics Guidelines. The Ethics Committee of the Showa University School of Medicine approved the study protocol. The study protocol is in compliance with the Declaration of Helsinki and current legal regulations in Japan. All procedures are in accordance with the ethical standards of the institutional and national committees responsible for human experimentation and with the Helsinki Declaration of 1964, as revised in 2013. Informed consent was obtained from all patients before being included in the study.

Data Availability. The data sets generated during and/or analyzed during the current study are available from the corresponding author on reasonable request.

Open Access. This article is distributed under the terms of the Creative Commons Attribution-NonCommercial 4.0 International
License (http://creativecommons.org/licenses/ by-nc/4.0/), which permits any noncommercial use, distribution, and reproduction in any medium, provided you give appropriate credit to the original author(s) and the source, provide a link to the Creative Commons license, and indicate if changes were made.

\section{REFERENCES}

1. Emerging Risk Factors Collaboration. Diabetes mellitus, fasting blood glucose concentration, and risk of vascular disease: a collaborative meta-analysis of 102 prospective studies. Lancet. 2010;375:2215-22. https://doi.org/10.1016/S01406736(10)60484-9.

2. Roglic G, Unwin N, Bennett PH, et al. The burden of mortality attributable to diabetes. Diabetes Care. 2005;28:2130-5. https://doi.org/10.2337/diacare. 28.9.2130.

3. Marso SP, Daniels GH, Brown-Frandsen K, LEADER Steering Committee, LEADER Trial Investigators, et al. Liraglutide and cardiovascular outcomes in type 2 diabetes. N Engl J Med. 2016;375:311-22.

4. Marso SP, Bain SC, Consoli A, et al. Semaglutide and cardiovascular outcomes in patients with Type 2 Diabetes. N Engl J Med. 2016;375:1834-44. https://doi.org/10.1056/NEJMoa1607141.

5. Ceriello A. The post-prandial state and cardiovascular disease: relevance to diabetes mellitus. Diabetes Metab Res Rev. 2000;16:125-32. https://doi. org/10.1002/(SICI)1520-7560(200003/04)16:2\% 3c125:AID-DMRR90\%3e3.0.CO;2-4.

6. Ohara M, Fukui T, Ouchi M, et al. Relationship between daily and day-to-day glycemic variability and increased oxidative stress in type 2 diabetes. Diabetes Res Clin Pract. 2016;122:62-70. https:// doi.org/10.1016/j.diabres.2016.09.025.

7. Torimoto K, Okada Y, Mori H, Tanaka Y. Relationship between fluctuations in glucose levels measured by continuous glucose monitoring and vascular endothelial dysfunction in type 2 diabetes mellitus. Cardiovasc Diabetol. 2013;12:1. https:// doi.org/10.1186/1475-2840-12-1.

8. Boye KS, Curtis SE, Lage MJ, Garcia-Perez LE. Associations between adherence and outcomes among older, type 2 diabetes patients: evidence from a Medicare Supplemental database. Patient Prefer Adherence. 2016;10:1573-81. https://doi.org/10. 2147/PPA.S107543. 
9. Yamamoto S, Hayashi T, Ohara M, et al. Comparison of liraglutide plus basal insulin and basal-bolus insulin therapy (BBIT) for glycemic control, body weight stability, and treatment satisfaction in patients treated using BBIT for type 2 diabetes without severe insulin deficiency: a randomized prospective pilot study. Diabetes Res Clin Pract. 2018;140:339-46. https://doi.org/10.1016/j.diabres. 2018.03.032.

10. Emoto M, Terauchi Y, Ozeki A, Oura T, Takeuchi M, Imaoka T. A 1-year safety study of dulaglutide in Japanese patients with type 2 diabetes on a single oral hypoglycemic agent: an open-label, nonrandomized, phase 3 trial. Endocr J. 2015;62:1101-14. https://doi.org/10.1507/endocrj.EJ15-0401.

11. Miyagawa J, Odawara M, Takamura T, Iwamoto N, Takita Y, Imaoka T. Once-weekly glucagon-like peptide-1 receptor agonist dulaglutide is non-inferior to once-daily liraglutide and superior to placebo in Japanese patients with type 2 diabetes: a 26-week randomized phase III study. Diabetes Obes Metab. 2015;17:974-83. https://doi.org/10.1111/ dom.12534.

12. Takase T, Nakamura A, Yamamoto C, et al. Improvement in treatment satisfaction after switching from liraglutide to dulaglutide in patients with type 2 diabetes: a randomized controlled trial. J Diabetes Investig. 2018. https://doi.org/10.1111/ jdi.12906 (Epub ahead of print).

13. Kuvin JT, Patel AR, Sliney KA, et al. Assessment of peripheral vascular endothelial function with finger arterial pulse wave amplitude. Am Heart J. 2003;146:168-74. https://doi.org/10.1016/S00028703(03)00094-2.

14. Service FJ, Molnar GD, Rosevear JW, Ackermann E, Gatewood LC, Taylor WF. Mean amplitude of glycemic excursions, a measure of diabetic instability. Diabetes. 1970;19:644-55. https://doi.org/10.2337/ diab.19.9.644.

15. Molnar GD, Taylor WF, Ho MM. Day-to-day variation of continuously monitored glycaemia: a further measure of diabetic instability. Diabetologia. 1972;8:342-8.

16. Monnier L, Colette C, Wojtusciszyn A, et al. Toward defining the threshold between low and high glucose variability in diabetes. Diabetes Care. 2017;40:832-8. https://doi.org/10.2337/dc16-1769.

17. Gerardi G, Usberti M, Martini G, et al. Plasma total antioxidant capacity in hemodialyzed patients and its relationship to other biomarkers of oxidative stress and lipid peroxidation. Clin Chem Lab Med. 2002;40:104-10. https://doi.org/10.1515/CCLM. 2002.019.
18. Cesarone MR, Belcaro G, Carratelli M, et al. A simple test to monitor oxidative stress. Int Angiol. 1999;18:127-30.

19. Schnedl WJ, Lahousen T, Wallner SJ, Krause R, Lipp RW. Silent hemoglobin variants and determination of HbA1c with the high resolution program of the HPLC HA-8160 hemoglobin analyzer. Clin Biochem. 2005;38:88-91. https://doi.org/10.1016/j. clinbiochem.2004.09.016.

20. Bradley C, Lewis KS. Measures of psychological well-being and treatment satisfaction developed from the responses of people with tablet-treated diabetes. Diabet Med. 1990;7:445-51. https://doi. org/10.1111/j.1464-5491.1990.tb01421.x.

21. Bradley $\mathrm{C}$. The diabetes treatment satisfaction questionnaire: DTSQ. In: Bradley C, editor. Handbook of psychology and diabetes: a guide to psychological measurement in diabetes research and practice. Chur: Harwood Academic; 1994. p. 111-32.

22. Inoue $\mathrm{K}$, Maeda $\mathrm{N}$, Kashine $\mathrm{S}$, et al. Short-term effects of liraglutide on visceral fat adiposity, appetite, and food preference: a pilot study of obese Japanese patients with type 2 diabetes. Cardiovasc Diabetol. 2011;10:109. https://doi.org/10.1186/ 1475-2840-10-109.

23. Lau KK, Wong YK, Chan YH, et al. Prognostic implications of surrogate markers of atherosclerosis in low to intermediate risk patients with type 2 diabetes. Cardiovasc Diabetol. 2012;11:101. https:// doi.org/10.1186/1475-2840-11-101.

24. Miyagawa J, Odawara M, Takamura T, Iwamoto N, Takita Y, Imaoka T. Once-weekly glucagon-like peptide-1 receptor agonist dulaglutide is non-inferior to once-daily liraglutide and superior to placebo in Japanese patients with type 2 diabetes: a 26-week randomized phase III study. Diabetes Obed Metab. 2015;17:974-83. https://doi.org/10.1111/ dom.12534.

25. Odawara M, Miyagawa J, Iwamoto N, Imaoka $T$, Takamuta T. Once-weekly glucagon-like peptide-1 receptor agonist dulaglutide significantly decreases glycated haemoglobin compared with once-daily liraglutide in Japanese patients with type 2 diabetes: 52 weeks of treatment in a randomized phase III study. Diabetes Obed Metab. 2016;18.249-57. https://doi.org/10.1111/dom.12602.

26. Jin SM, Kim TH, Bae JC, Hur KY, Lee MS, Lee MK. Clinical factors associated with absolute and relative measures of glycemic variability determined by continuous glucose monitoring: an analysis of 480 subjects. Diabetes Res Clin Pract. 2014;104:266-72. https://doi.org/10.1016/j.diabres.2014.02.003. 
27. Ohara M, Nagaike H, Goto S, et al. Improvements of ambient hyperglycemia and glycemic variability are associated with reduction in oxidative stress for patients with type 2 diabetes. Diabetes Res Clin Pract. 2018;139:253-61. https://doi.org/10.1016/j. diabres.2018.02.017.

28. Ceriello A, Novials A, Ortega E, et al. Glucagon-like peptide 1 reduces endothelial dysfunction, inflammation, and oxidative stress induced by both hyperglycemia and hypoglycemia in type 1 diabetes. Diabetes Care. 2013;36:2346-50. https://doi. org/10.2337/dc12-2469.

29. Ceriello A, Novials A, Canivell S, et al. Simultaneous GLP-1 and insulin administration acutely enhances their vasodilatory, antiinflammatory, and antioxidant action in type 2 diabetes. Diabetes Care. 2014;37:1938-43. https://doi.org/10.2337/dc132618.

30. Dandona P, Chaudhuri A, Ghanim H, Mohanty P. Insulin as an anti-inflammatory and antiatherogenic modulator. J Am Coll Cardiol.
2009;53:S14-20. 2008.10.038.

https://doi.org/10.1016/j.jacc.

31. Oeseburg H, de Boer RA, Buikema $H$, van der Harst $\mathrm{P}$, van Gilst WH, Silljé HH. Glucagon-like peptide 1 prevents reactive oxygen species-induced endothelial cell senescence through the activation of protein kinase A. Arterioscler Thromb Vasc Biol. 2010;30:1407-14. https://doi.org/10.1161/ ATVBAHA.110.206425.

32. Klonoff DC, Buse JB, Nielsen LL, et al. Exenatide effects on diabetes, obesity, cardiovascular risk factors and hepatic biomarkers in patients with type 2 diabetes treated for at least 3 years. Curr Med Res Opin. 2008;24:275-86. https://doi.org/10.1185/ $030079908 \times 253870$.

33. Yang $\mathrm{SH}, \mathrm{Xu} \mathrm{RX}, \mathrm{Cui} \mathrm{CJ}$, et al. Liraglutide downregulates hepatic LDL receptor and PCSK9 expression in HepG2 cells and $\mathrm{db} / \mathrm{db}$ mice through a HNF1a dependent mechanism. Cardiovasc Diabetol. 2018;17:48. https://doi.org/10.1186/s12933-0180689-9. 